Bull. Austral. Math. Soc.

26D15, 41A55

VOL. 67 (2003) [257-266]

\title{
SHARP BOUNDS OF ČEBYŠEV FUNCTIONAL FOR STIELTJES INTEGRALS AND APPLICATIONS
}

\section{S.S. DRAGOMIR}

Sharp bounds of the Čebyšev functional for the Stieltjes integrals similar to the Grüss one and applications for quadrature rules are given.

\section{INTRODUCTION}

Consider the weighted $\check{C}$ ebyšev functional

(1.1)

$$
\begin{aligned}
T_{w}(f, g):=\frac{1}{\int_{a}^{b} w(t) d t} & \int_{a}^{b} w(t) f(t) g(t) d t \\
& -\frac{1}{\int_{a}^{b} w(t) d t} \int_{a}^{b} w(t) f(t) d t \cdot \frac{1}{\int_{a}^{b} w(t) d t} \int_{a}^{b} w(t) g(t) d t
\end{aligned}
$$

where $f, g, w:[a, b] \rightarrow \mathbb{R}$ and $w(t) \geqslant 0$ for almost every $t \in[a, b]$ are measurable functions such that the involved integrals exist and $\int_{a}^{b} w(t) d t>0$.

In $[1]$, the authors obtained, among others, the following inequalities:

$$
\begin{aligned}
& \left|T_{w}(f, g)\right| \\
& \leqslant \frac{1}{2}(M-m) \frac{1}{\int_{a}^{b} w(t) d t} \int_{a}^{b} w(t)\left|g(t)-\frac{1}{\int_{a}^{b} w(s) d s} \int_{a}^{b} w(s) g(s) d s\right| d t \\
& \leqslant \frac{1}{2}(M-m)\left[\frac{1}{\int_{a}^{b} w(t) d t} \int_{a}^{b} w(t)\right. \\
& \left.\quad \times\left|g(t)-\frac{1}{\int_{a}^{b} w(s) d s} \int_{a}^{b} w(s) g(s) d s\right|^{p} d t\right]^{1 / p}(p>1) \\
& \leqslant \frac{1}{2}(M-m) e s s \sup _{t \in[a, b]}\left|g(t)-\frac{1}{\int_{a}^{b} w(s) d s} \int_{a}^{b} w(s) g(s) d s\right|
\end{aligned}
$$

provided

$$
-\infty<m \leqslant f(t) \leqslant M<\infty \text { for almost every } t \in[a, b]
$$

Received 2nd September, 2002

Copyright Clearance Centre, Inc. Serial-fee code: 0004-9727/03 \$A2.00+0.00. 
and the corresponding integrals are finite. The constant $1 / 2$ is sharp in all the inequalities in (1.2) in the sense that it cannot be replaced by a smaller constant.

In addition, if

$$
-\infty<n \leqslant g(t) \leqslant N<\infty \text { for almost every } t \in[a, b],
$$

then the following refinement of the celebrated Grüss inequality is obtained:

$$
\begin{aligned}
& \left|T_{w}(f, g)\right| \\
& \quad \leqslant \frac{1}{2}(M-m) \frac{1}{\int_{a}^{b} w(t) d t} \int_{a}^{b} w(t)\left|g(t)-\frac{1}{\int_{a}^{b} w(s) d s} \int_{a}^{b} w(s) g(s) d s\right| d t \\
& \quad \leqslant \frac{1}{2}(M-m)\left[\frac{1}{\int_{a}^{b} w(t) d t} \int_{a}^{b} w(t)\right. \\
& \left.\quad \times\left|g(t)-\frac{1}{\int_{a}^{b} w(s) d s} \int_{a}^{b} w(s) g(s) d s\right|^{2} d t\right]^{1 / 2} \\
& \quad \leqslant \frac{1}{4}(M-m)(N-n) .
\end{aligned}
$$

Here, the constants $1 / 2$ and $1 / 4$ are also sharp in the sense mentioned above.

In this paper, we extend the above results to Riemann-Stieltjes integrals. A quadrature formula is also considered.

For this purpose, we introduce the following Cebyšev functional for the Stieltjes integral

$$
\begin{aligned}
T(f, g ; u):=\frac{1}{u(b)-u(a)} \int_{a}^{b} f(t) g(t) d u(t) \\
\quad-\frac{1}{u(b)-u(a)} \int_{a}^{b} f(t) d u(t) \cdot \frac{1}{u(b)-u(a)} \int_{a}^{b} g(t) d u(t),
\end{aligned}
$$

where $f, g \in C[a, b]$ (are continuous on $[a, b]$ ) and $u \in B V[a, b]$ (is of bounded variation on $[a, b])$ with $u(b) \neq u(a)$.

For some recent inequalities for Stieltjes integral see [2]-[5].

\section{The Results}

The following result holds.

THEOREM 1. Let $f, g:[a, b] \rightarrow \mathbb{R}$ be continuous on $[a, b]$ and $u:[a, b] \rightarrow \mathbb{R}$ with $u(a) \neq u(b)$. Assume also that there exists the real constants $m, M$ such that

$$
m \leqslant f(t) \leqslant M \text { for each } t \in[a, b] .
$$


If $u$ is of bounded variation on $[a, b]$, then we have the inequality

$$
\begin{aligned}
&|T(f, g ; u)| \leqslant \frac{1}{2}(M-m) \frac{1}{|u(b)-u(a)|} \\
& \times\|\| g-\frac{1}{u(b)-u(a)} \int_{a}^{b} g(s) d u(s) \|_{\infty} \bigvee_{a}^{b}(u),
\end{aligned}
$$

where $\bigvee_{a}^{b}(u)$ denotes the total variation of $u$ in $[a, b]$. The constant $1 / 2$ is sharp, in the sense that it cannot be replaced by a smaller constant.

Proof: It is easy to see, by simple computation with the Stieltjes integral, that the following equality

$$
\begin{aligned}
T(f, g ; u)=\frac{1}{u(b)-u(a)} \int_{a}^{b}[f(t) & \left.-\frac{m+M}{2}\right] \\
& \times\left[g(t)-\frac{1}{u(b)-u(a)} \int_{a}^{b} g(s) d u(s)\right] d u(t)
\end{aligned}
$$

holds.

Using the known inequality

$$
\left|\int_{a}^{b} p(t) d v(t)\right| \leqslant \sup _{t \in[a, b]}|p(t)| \bigvee_{a}^{b}(v)
$$

provided $p \in C[a, b]$ and $v \in B V[a, b]$, we have, by (2.3), that

$$
\begin{aligned}
|T(f, g ; u)| & \leqslant \sup _{t \in[a, b]}\left|\left[f(t)-\frac{m+M}{2}\right]\left[g(t)-\frac{1}{u(b)-u(a)} \int_{a}^{b} g(s) d u(s)\right]\right| \\
& \cdot \frac{1}{|u(b)-u(a)|} \bigvee_{a}^{b}(u) \\
& \left(\text { since }\left|f(t)-\frac{m+M}{2}\right| \leqslant \frac{M-m}{2} \text { for any } t \in[a, b]\right) \\
& \leqslant \frac{M-m}{2}\left\|g-\frac{1}{u(b)-u(a)} \int_{a}^{b} g(s) d u(s)\right\|_{\infty} \cdot \frac{1}{|u(b)-u(a)|} \bigvee_{a}^{b}(u)
\end{aligned}
$$

and the inequality (2.2) is proved.

To prove the sharpness of the constant $1 / 2$ in the inequality (2.2), we assume that it holds with a constant $C>0$, that is,

$$
\begin{aligned}
& |T(f, g ; u)| \leqslant C(M-m) \frac{1}{|u(b)-u(a)|} \\
& \quad \times\left\|g-\frac{1}{u(b)-u(a)} \int_{a}^{b} g(s) d u(s)\right\|_{\infty} \bigvee_{a}^{b}(u) .
\end{aligned}
$$


Let us consider the functions $f=g, f:[a, b] \rightarrow \mathbb{R}, f(t)=t, t \in[a, b]$ and $u:[a, b] \rightarrow \mathbb{R}$ given by

$$
u(t)= \begin{cases}-1 & \text { if } t=a, \\ 0 & \text { if } t \in(a, b), \\ 1 \quad \text { if } t=b .\end{cases}
$$

Then $f, g$ are continuous on $[a, b], u$ is of bounded variation on $[a, b]$ and

$$
\begin{aligned}
\frac{1}{u(b)-u(a)} \int_{a}^{b} f(t) g(t) d u(t) & =\frac{1}{2} \int_{a}^{b} t^{2} d u(t) \\
& =\frac{1}{2}\left[\left.t^{2} u(t)\right|_{a} ^{b}-2 \int_{a}^{b} t u(t) d t\right] \\
& =\frac{b^{2}+a^{2}}{2}, \\
\frac{1}{u(b)-u(a)} \int_{a}^{b} f(t) d u(t) & =\frac{1}{u(b)-u(a)} \int_{a}^{b} g(t) d u(t) \\
& =\frac{1}{2} \int_{a}^{b} t d u(t) \\
& =\frac{1}{2}\left[\left.t u(t)\right|_{a} ^{b}-\int_{a}^{b} u(t) d t\right] \\
& =\frac{b+a}{2}, \\
\| g-\frac{1}{u(b)-u(a)} \int_{a}^{b} g(s) d u(s) & \|_{\infty}=\sup _{t \in[a, b]}\left|t-\frac{a+b}{2}\right|=\frac{b-a}{2}
\end{aligned}
$$

and

$$
\bigvee_{a}^{b}(u)=2, \quad M=b, \quad m=a .
$$

Inserting these values in (2.5), we get

$$
\left|\frac{a^{2}+b^{2}}{2}-\frac{(a+b)^{2}}{4}\right| \leqslant C(b-a) \cdot \frac{1}{2} \cdot \frac{(b-a)}{2} \cdot 2,
$$

giving $C \geqslant 1 / 2$, and the theorem is thus proved.

The corresponding result for a monotonic function $u$ is incorporated in the following theorem.

THEOREM 2. Assume that $f$ and $g$ are as in Theorem 1. If $u:[a, b] \rightarrow \mathbb{R}$ is monotonic nondecreasing on $[a, b]$, then one has the inequality:

$$
\begin{aligned}
& |T(f, g ; u)| \leqslant \frac{1}{2}(M-m) \frac{1}{u(b)-u(a)} \\
& \times \int_{a}^{b}\left|g(t)-\frac{1}{u(b)-u(a)} \int_{a}^{b} g(s) d u(s)\right| d u(t) .
\end{aligned}
$$


The constant $1 / 2$ is sharp in the sense that it cannot be replaced by a smaller constant.

PROOF: Using the known inequality

$$
\left|\int_{a}^{b} p(t) d v(t)\right| \leqslant \int_{a}^{b}|p(t)| d v(t)
$$

provided $p \in C[a, b]$ and $v$ is a monotonic nondecreasing function on $[a, b]$, we have (by the use of equality (2.3)) that

$$
\begin{aligned}
|T(f, g ; u)| \leqslant \frac{1}{u(b)-u(a)} \int_{a}^{b}\left|f(t)-\frac{m+M}{2}\right| & \\
\cdot \quad & \times\left|g(t)-\frac{1}{u(b)-u(a)} \int_{a}^{b} g(s) d u(s)\right| d u(t) \\
& \leqslant \frac{1}{2}(M-m) \frac{1}{u(b)-u(a)} \int_{a}^{b}\left|g(t)-\frac{1}{u(b)-u(a)} \int_{a}^{b} g(s) d u(s)\right| d u(t) .
\end{aligned}
$$

Now, assume that the inequality (2.7) holds with a constant $D>0$, instead of $1 / 2$, that is,

$$
\begin{aligned}
& |T(f, g ; u)| \leqslant D(M-m) \frac{1}{u(b)-u(a)} \\
& \times \int_{a}^{b}\left|g(t)-\frac{1}{u(b)-u(a)} \int_{a}^{b} g(s) d u(s)\right| d u(t) .
\end{aligned}
$$

If we choose the same function as in the proof of Theorem 1 , we observe that $f, g$ are continuous and $u$ is monotonic nondecreasing on $[a, b]$. Then, for these functions, we have

$$
\begin{gathered}
T(f, g ; u)=\frac{a^{2}+b^{2}}{2}-\frac{(a+b)^{2}}{4}=\frac{(b-a)^{2}}{4} \\
\int_{a}^{b}\left|g(t)-\frac{1}{u(b)-u(a)} \int_{a}^{b} g(s) d u(s)\right| d u(t) \\
=\int_{a}^{b}\left|t-\frac{a+b}{2}\right| d u(t) \\
=\int_{a}^{(a+b) / 2}\left(\frac{a+b}{2}-t\right) d u(t)+\int_{(a+b) / 2}^{b}\left(t-\frac{a+b}{2}\right) d u(t) \\
=\left.\left[u(t)\left(\frac{a+b}{2}-t\right)\right]\right|_{a} ^{(a+b) / 2}+\int_{a}^{(a+b) / 2} u(t) d t \\
=b-a,\left.\left[\left(t-\frac{a+b}{2}\right) u(t)\right]\right|_{(a+b) / 2} ^{b} \int_{(a+b) / 2}^{b} u(t) d t
\end{gathered}
$$


and then, by (2.9) we get

$$
\frac{(b-a)^{2}}{4} \leqslant D(b-a) \frac{1}{2}(b-a)
$$

giving $D \geqslant 1 / 2$, and the theorem is completely proved.

The case when $u$ is a Lipschitzian function is embodied in the following theorem.

TheOREM 3. Assume that $f, g:[a, b] \rightarrow \mathbb{R}$ are Riemann integrable functions on $[a, b]$ and $f$ satisfies the condition (2.1). If $u:(a, b) \rightarrow \mathbb{R}(u(b) \neq u(a))$ is Lipschitzian with the constant $L$, then we have the inequality

$$
\begin{aligned}
&|T(f, g ; u)| \leqslant \frac{1}{2} L(M-m) \frac{1}{|u(b)-u(a)|} \\
& \times \int_{a}^{b}\left|g(t)-\frac{1}{u(b)-u(a)} \int_{a}^{b} g(s) d u(s)\right| d t .
\end{aligned}
$$

The constant $1 / 2$ cannot be replaced by a smaller constant.

PROOF: It is well known that if $p:[a, b] \rightarrow \mathbb{R}$ is Riemann integrable on $[a, b]$ and $v:[a, b] \rightarrow \mathbb{R}$ is Lipschitzian with the constant $L$, then the Riemann-Stieltjes integral $\int_{a}^{b} p(t) d v(t)$ exists and

$$
\left|\int_{a}^{b} p(t) d v(t)\right| \leqslant L \int_{a}^{b}|p(t)| d t .
$$

Using this fact and the identity (2.3), we deduce

$$
\begin{aligned}
|T(f, g ; u)| & \leqslant \frac{L}{|u(b)-u(a)|} \int_{a}^{b}\left|f(t)-\frac{m+M}{2}\right| \\
\times\left|g(t)-\frac{1}{u(b)-u(a)} \int_{a}^{b} g(s) d u(s)\right| d t & \leqslant \frac{1}{2}(M-m) \frac{L}{|u(b)-u(a)|} \int_{a}^{b}\left|g(t)-\frac{1}{u(b)-u(a)} \int_{a}^{b} g(s) d u(s)\right| d t
\end{aligned}
$$

and the inequality $(2.10)$ is proved.

Now, assume that (2.10) holds with a constant $E>0$ instead of $1 / 2$, that is,

$$
\begin{aligned}
&|T(f, g ; u)| \leqslant E L(M-m) \frac{1}{|u(b)-u(a)|} \\
& \quad \times \int_{a}^{b}\left|g(t)-\frac{1}{u(b)-u(a)} \int_{a}^{b} g(s) d u(s)\right| d t .
\end{aligned}
$$

Consider the function $f=g, f:[a, b] \rightarrow \mathbb{R}$ with

$$
f(t)= \begin{cases}-1 & \text { if } t \in\left[a, \frac{a+b}{2}\right] \\ 1 & \text { if } t \in\left(\frac{a+b}{2}, b\right]\end{cases}
$$


and $u:[a, b] \rightarrow \mathbb{R}, u(t)=t$. Then, obviously, $f$ and $g$ are Riemann integrable on $[a, b]$ and $u$ is Lipschitzian with the constant $L=1$.

Since

$$
\begin{gathered}
\frac{1}{u(b)-u(a)} \int_{a}^{b} f(t) g(t) d u(t)=\frac{1}{b-a} \int_{a}^{b} d t=1, \\
\frac{1}{u(b)-u(a)} \int_{a}^{b} f(t) d u(t)=\frac{1}{u(b)-u(a)} \int_{a}^{b} g(t) d u(t)=0, \\
\int_{a}^{b}\left|g(t)-\frac{1}{u(b)-u(a)} \int_{a}^{b} g(s) d u(s)\right| d t=\int_{a}^{b} d t=b-a
\end{gathered}
$$

and

$$
M=1, \quad m=1
$$

then, by (2.12), we deduce $E \geqslant 1 / 2$, and the theorem is completely proved.

\section{A Quadrature Formula}

Let us consider the partition of the interval $[a, b]$ given by

$$
I_{n}: a=x_{0}<x_{1}<\cdots<x_{n-1}<x_{n}=b .
$$

Denote $v\left(I_{n}\right):=\max \left\{h_{i} \mid i=\overline{0, n-1}\right\}$ where $h_{i}:=x_{i+1}-x_{i}, i=\overline{0, n-1}$.

If $f:[a, b] \rightarrow \mathbb{R}$ is continuous on $[a, b]$ and if we define

$$
\begin{aligned}
M_{i} & :=\sup _{t \in\left[x_{i}, x_{i+1}\right]} f(t), m_{i}:=\inf _{t \in\left[x_{i}, x_{i+1}\right]} f(t), \text { and } \\
v\left(f, I_{n}\right) & =\max _{i=\overline{0, n-1}}\left(M_{i}-m_{i}\right),
\end{aligned}
$$

then, obviously, by the continuity of $f$ on $[a, b]$, for any $\varepsilon>0$, we may find a division $I_{n}$ with norm $v\left(I_{n}\right)<\delta$ such that $v\left(f, I_{n}\right)<\varepsilon$.

Consider now the quadrature rule

$$
S_{n}\left(f, g ; u, I_{n}\right):=\sum_{i=0}^{n-1} \frac{1}{u\left(x_{i+1}\right)-u\left(x_{i}\right)} \int_{x_{i}}^{x_{i+1}} f(t) d u(t) \cdot \int_{x_{i}}^{x_{i+1}} g(t) d u(t)
$$

provided $f, g \in C[a, b], u \in B V[a, b]$ and $u\left(x_{i+1}\right) \neq u\left(x_{i}\right), i=0, \ldots, n-1$.

We may now state the following result in approximating the Stieltjes integral

$$
\int_{a}^{b} f(t) g(t) d u(t)
$$

THEOREM 4. Let $f, g \in C[a, b]$ and $u \in B V[a, b]$. If $I_{n}$ is a division of the interval $[a, b]$ and $u\left(x_{i+1}\right) \neq u\left(x_{i}\right), i=0, \ldots, n-1$, then we have:

$$
\int_{a}^{b} f(t) g(t) d u(t)=S_{n}\left(f, g ; u, I_{n}\right)+R_{n}\left(f, g ; u, I_{n}\right)
$$


where $S_{n}\left(f, g ; u, I_{n}\right)$ is as defined in (3.2) and the remainder $R_{n}\left(f, g ; u, I_{n}\right)$ satisfies the estimate

$$
\begin{aligned}
&\left|R_{n}\left(f, g ; u, I_{n}\right)\right| \leqslant \frac{1}{2} v\left(f, I_{n}\right) \\
& \times \max _{i=0, n-1}\left\|g-\frac{1}{u\left(x_{i+1}\right)-u\left(x_{i}\right)} \int_{x_{i}}^{x_{i+1}} g(s) d u(s)\right\|_{\left[x_{i}, x_{i+1}, \infty\right.} \bigvee_{a}^{b}(u) .
\end{aligned}
$$

The constant $1 / 2$ is sharp in (3.4) in the sense that it cannot be replaced by a smaller constant.

Proof: Applying the inequality (2.2) on the intervals $\left[x_{i}, x_{i+1}\right], i=0, \ldots, n-1$, we have

$$
\begin{aligned}
& \left|\int_{x_{i}}^{x_{i+1}} f(t) g(t) d u(t)-\frac{1}{u\left(x_{i+1}\right)-u\left(x_{i}\right)} \int_{x_{i}}^{x_{i+1}} f(t) d u(t) \cdot \int_{x_{i}}^{x_{i+1}} g(t) d u(t)\right| \\
& \quad \leqslant \frac{1}{2}\left(M_{i}-m_{i}\right) \sup _{t \in\left[x_{i}, x_{i+1}\right]}\left|g(t)-\frac{1}{u\left(x_{i+1}\right)-u\left(x_{i}\right)} \int_{x_{i}}^{x_{i+1}} g(s) d u(s)\right| \bigvee_{x_{i}}^{x_{i+1}}(u) .
\end{aligned}
$$

Summing the inequalities (3.5) over $i$ from 0 to $n-1$, and using the generalised triangle inequality, we have

$$
\begin{aligned}
& \left|R_{n}\left(f, g ; u, I_{n}\right)\right| \\
& \leqslant \frac{1}{2} \sum_{i=0}^{n-1}\left(M_{i}-m_{i}\right)\left\|g-\frac{1}{u\left(x_{i+1}\right)-u\left(x_{i}\right)} \int_{x_{i}}^{x_{i+1}} g(s) d u(s)\right\|_{\left[x_{i}, x_{i+1}\right], \infty} \\
& \times \bigvee_{x_{i}}^{x_{i+1}}(u) \\
& \leqslant \frac{1}{2} v\left(f, I_{n}\right) \max _{i=\overline{0}, n-1}\left\|g-\frac{1}{u\left(x_{i+1}\right)-u\left(x_{i}\right)} \int_{x_{i}}^{x_{i+1}} g(s) d u(s)\right\|_{\left[x_{i}, x_{i+1}\right], \infty} \\
& \times \sum_{i=0}^{n-1} \bigvee_{x_{i}}^{x_{i+1}}(u) \\
& =\frac{1}{2} v\left(f, I_{n}\right) \max _{i=\overline{0}, n-1}\left\|g-\frac{1}{u\left(x_{i+1}\right)-u\left(x_{i}\right)} \int_{x_{i}}^{x_{i+1}} g(s) d u(s)\right\|_{\left[x_{i}, x_{i+1}\right], \infty} \\
& \times \bigvee_{a}^{b}(u)
\end{aligned}
$$

and the estimate (3.4) is obtained.

REMARK 1. Similar results may be stated for either $u$ monotonic or Lipschitzian. We omit the details. 


\section{Some Particular Cases}

For $f, g, w:[a, b] \rightarrow \mathbb{R}$, integrable and with the property that $\int_{a}^{b} w(t) d t \neq 0$, reconsider the weighted Čbyšev functional

$$
\begin{aligned}
T_{w}(f, g):=\frac{1}{\int_{a}^{b} w(t) d t} & \int_{a}^{b} w(t) f(t) g(t) d t \\
& -\frac{1}{\int_{a}^{b} w(t) d t} \int_{a}^{b} w(t) f(t) d t \cdot \frac{1}{\int_{a}^{b} w(t) d t} \int_{a}^{b} w(t) g(t) d t
\end{aligned}
$$

1. If $f, g, w:[a, b] \rightarrow \mathbb{R}$ are continuous and there exists the real constants $m, M$ such that

$$
m \leqslant f(t) \leqslant M \text { for each } t \in[a, b]
$$

then one has the inequality

$$
\begin{aligned}
&\left|T_{w}(f, g)\right| \leqslant \frac{1}{2}(M-m) \frac{1}{\left|\int_{a}^{b} w(s) d s\right|} \\
& \times\left\|g-\frac{1}{\int_{a}^{b} w(s) d s} \int_{a}^{b} g(s) w(s) d s\right\|_{[a, b], \infty} \int_{a}^{b}|w(s)| d s .
\end{aligned}
$$

The proof follows by Theorem 1 on choosing $u(t)=\int_{a}^{t} w(s) d s$.

2. If $f, g, w$ are as in $\mathbf{1}$ and $w(s) \geqslant 0$ for $s \in[a, b]$, then one has the inequality

$$
\begin{aligned}
& \left|T_{w}(f, g)\right| \leqslant \frac{1}{2}(M-m) \frac{1}{\int_{a}^{b} w(s) d s} \\
& \quad \times \int_{a}^{b}\left|g(t)-\frac{1}{\int_{a}^{b} w(s) d s} \int_{a}^{b} g(s) w(s) d s\right| w(s) d s .
\end{aligned}
$$

The proof follows by Theorem 2 on choosing $u(t)=\int_{a}^{t} w(s) d s$.

3. If $f, g$ are Riemann integrable on $[a, b]$ and $f$ satisfies (4.2), and $w$ is continuous on $[a, b]$, then one has the inequality

$$
\begin{aligned}
\left|T_{w}(f, g)\right| \leqslant \frac{1}{2}\|w\|_{[a, b], \infty}(M-m) & \frac{1}{\left|\int_{a}^{b} w(s) d s\right|} \\
& \times \int_{a}^{b}\left|g(t)-\frac{1}{\int_{a}^{b} w(s) d s} \int_{a}^{b} g(s) w(s) d s\right| d s
\end{aligned}
$$

The proof follows by Theorem 3 on choosing $u(t)=\int_{a}^{t} w(s) d s$. 


\section{REFERENCES}

[1] P. Cerone and S.S. Dragomir, 'A refinement of the Grüss inequality and applications', J. Inequal. Pure Appl. Math. 5 (2002). Article 14 [ONLINE: http://rgmia.vu.edu.au/v5n2.html].

[2] S.S. Dragomir, 'On the Ostrowski's inequality for Riemann-Stieltjes integral and applications', Korean J. Comput. and Appl. Math. 7 (2000), 611-627.

[3] S.S. Dragomir, 'Some inequalities for Riemann-Stieltjes integral and applications', in $O p$ timisation and Related Topics, (A. Rubinov, Editor), Appl. Optim. 47 (Kluwer Academic Publishers, Dorchrecht, 2000), pp. 197-235.

[4] S.S. Dragomir, 'Some inequalities of midpoint and trapezoid type for the Riemann-Stieltjes integral', Nonlinear Anal. 47 (2001), 2333-234.

[5] S.S. Dragomir, 'On the Ostrowski inequality for Riemann-Stieltjes integral where $f$ is of Hölder type and $u$ is of bounded variation and applications', J. KSIAM 5 (2001), 35-45.

School of Computer Science and Mathematics

Victoria University of Technology

P.O. Box 14428

MCMC 8001, Vic.

Australia

e-mail: sever@matilda.vu.edu.au

urladdr: http://rgmia.vu.edu.au/SSDragomirWeb.html 